\title{
RE: The effect of atropine in preventing catheter-related pain and discomfort in patients undergoing transurethral resection due to bladder tumor: a prospective, randomized, controlled study
}

\author{
Joon-Ho Lee \\ Department of Anesthesiology and Pain Medicine, Soonchunhyang University Bucheon Hospial, Bucheon, Korea
}

Received May 14, 2020, Revised May 18, 2020, Accepted May 20, 2020

Handling Editor: Francis Sahngun Nahm

Correspondence

Joon-Ho Lee

Department of Anesthesiology and Pain Medicine, Soonchunhyang University Bucheon Hospital, 170 Jomaru-ro, Wonmi-gu, Bucheon 14584, Korea Tel: +82-32-621-5114, Fax: +82-32-621-5114, E-mail: anpjuno@schmc.ac.kr

\section{LETTER TO THE EDITOR}

Recently, Șahiner et al. [1] reported a novel study demonstrating the positive effect of intraoperative atropine in the prevention of postoperative catheter-related bladder discomfort (CRBD) following transurethral resection of bladder tumor (TURBT). The aim of this study was to determine the anticholinergic effect of atropine on suppression of bladder contraction that is known to be related to CRBD. This study had several valuable points for use in routine clinical practice, namely, the clinical importance of CRBD, the feasibility of the use of atropine treatment with safety concerns, and replacing or overcoming the use of other antimuscarinic anticholinergics such as ketamine, tramadol, butylscopolamine, oxybutynin, solifenacin, tolterodine, fesoterodine, imidafenacin, and trospium.

CRBD is one of the most distressing symptoms experienced during recovery from anesthesia due to the involuntary bladder contraction caused by the indwelling catheter. Although there have been no large epidemiologic studies investigating the prevalence of CRBD, it is an important clinical entity with a prevalence ranging from $47 \%$ to $90 \%$
[2]. Moreover, this symptomatic entity can cause emergence agitation during the early postoperative recovery period [3].

Atropine is a cheap and easy-to-use drug with a long history of safe usage. According to Șahiner et al. [1], atropine could be an attractive treatment option to treat CRBD without any significant adverse events. The previously used anticholinergics such as butylscopolamine oxybutynin, solifenacin, tolterodine, fesoterodine, imidafenacin, and trospium chloride showed limited effectiveness but caused prominent side effects such as dry mouth and constipation. Opioids and non-steroidal anti-inflammatory drugs could not be used to reduce CRBD pain symptoms because of their different pathophysiologic mechanisms in postoperative pain management. The pathophysiologic mechanism of CRBD causing distress or discomfort is similar to that of an overactive bladder that could be treated with anticholinergics by blocking muscarinic receptors.

Atropine is a safe anticholinergic agent for intraoperative administration because it is the prototype of the antimuscarinic anticholinergic drugs that block muscarinic receptors in the bladder. Other anticholinergics, especially those reported for the treatment of overactive bladder, are (a) This is an open-access article distributed under the terms of the Creative Commons Attribution Non-Commercial License (http://creativecommons.org/licenses/by-nc/4.0/), which permits unrestricted non-commercial use, distribution, and reproduction in any medium, provided the original work is properly cited.

(c) The Korean Pain Society, 2020
Author contributions: Joon-Ho Lee: Writing/manuscript preparation. 
not fit for systemic use during anesthesia. Furthermore, atropine is also an inexpensive and easily accessible treatment option; however, in order to apply the results of this study in clinical practice, some important issues need to be clarified, despite the aforementioned advantages of this study. Although atropine is a generally considered a safe drug, clinicians must thoroughly look at its side effects, including mild vagal excitatory effects in therapeutic doses, and its ability to cross the blood-brain barrier and cause central excitation in high doses that can manifest as restlessness, delirium, disorientation, and hallucination [4]. Secondly, the gap in postoperative numeric rating scale (NRS) scores at $0 \mathrm{~h}$ between the two treatment groups were found to be too high, which does not appear realistic in practice. Moreover, the postoperative NRS score of Group 1 at $24 \mathrm{~h}$ was reported as $0.0 \pm 0.0$ which is quite improbable because there should be some discomfort with an indwelling urethral catheter, although minimal. In the Materials and Methods section, there is no information about the indwelling duration of the urethral catheter; however, traditionally, the urethral catheter is left for at least 2 to 5 days for safe healing of the resected bladder. Furthermore, the authors have not mentioned postoperative urinary retention; perhaps the authors need to inform about the incidence of postoperative urinary retention, especially in cases with simple TURBT as it allows early removal of the urethral catheter on the postoperative day. Dreijer et al. [5] reported the prevalence of postoperative urinary retention in 334 consecutive adult patients who underwent surgery as $4.8 \%$. They have also reported on significant risk factors, such as diabetes mellitus (OR, 5.9; 95\% CI, 1.760-19.882) and atropine administration during surgery (OR, 5.9; $95 \%$ CI, 1.005-34.680) that need to be considered. Tammela et al. [6] have also reported intraoperative administration of sympathomimetic and anticholinergic medication as a risk factor for postoperative urinary retention. Lastly, other significant variables, namely, resected tumor volume, intravesical chemotherapy, and continuous irrigation, that could affect involuntary bladder contraction, should also be considered.

Considering the clinical importance of CRBD, efforts to prevent or minimize CRBD have to be expanded into the entire surgery program, and should not be just limited to TURBT. In a large observational study by $\mathrm{Li}$ et al. [7], the authors reported that moderate and severe CRBD were significantly related to the conduction of open abdominal surgery and a previous history of catheterization. Recently, other treatment options such as intraoperative administration of lidocaine or magnesium, or a single intravenous injection of parecoxib and pudendal nerve block have been introduced to prevent CRBD. Considering the high prevalence of CRBD, efforts and strategies need to be targeted toward the standardization of catheterizationrequiring surgical procedures.

\section{CONFLICT OF INTEREST}

No potential conflict of interest relevant to this article was reported.

\section{FUNDING}

This work was supported by Soonchunhyang University Research Fund.

\section{ORCID}

Joon-Ho Lee, https://orcid.org/0000-0002-6384-7263

\section{REFERENCES}

1. Șahiner Y, Yağan Ö, Akdağlı Ekici A, Ekici M, Demir E. The effect of atropine in preventing catheter-related pain and discomfort in patients undergoing transurethral resection due to bladder tumor; prospective randomized, controlled study. Korean J Pain 2020; 33: 176-82.

2. Bai Y, Wang X, Li X, Pu C, Yuan H, Tang Y, et al. Management of catheter-related bladder discomfort in patients who underwent elective surgery. J Endourol 2015; 29: 640-9.

3. Kim HC, Kim E, Jeon YT, Hwang JW, Lim YJ, Seo JH, et al. Postanaesthetic emergence agitation in adult patients after general anaesthesia for urological surgery. J Int Med Res 2015; 43: 226-35.

4. Toft P, Rømer UD. Glycopyrrolate compared with atropine in association with ketamine anaesthesia. Acta Anaesthesiol Scand 1987; 31: 438-40.

5. Dreijer B, Møller MH, Bartholdy J. Post-operative urinary retention in a general surgical population. Eur J Anaesthesiol 2011; 28: 190-4.

6. Tammela T, Kontturi M, Lukkarinen O. Postoperative urinary retention. I. Incidence and predisposing factors. Scand J Urol Nephrol 1986; 20: 197-201.

7. Li C, Liu Z, Yang F. Predictors of catheter-related bladder discomfort after urological surgery. J Huazhong Univ Sci Technolog Med Sci 2014; 34: 559-62. 